Open Access

\title{
A Novel Method for Forest Leaf Area Index Inversion Using Lidar Data
}

\author{
Huang Zuowei ${ }^{*}, 1$, Huang Yuanjiang ${ }^{1}$ and Guo Yadong ${ }^{2}$ \\ ${ }^{1}$ School of Architecture and Urban Planning, Hunan University of Technology, Zhuzhou 412008, China; ${ }^{2}$ School of \\ Geosciences and Information-Physics, Central South University, Changsha 410083, China
}

\begin{abstract}
Leaf area index (LAI) is one of the key ecological parameters and can be widely used in the growth situation of vegetation and environmental assessment. LiDAR is a new emerging active remote sensing technology in recent years, which can measure both the vertical and horizontal structure of forested areas effectively with high precision. Meanwhile, with the LiDAR data greatly enriches the observations of land surface targets, which makes it possible to estimate forest structural parameters accurately. Aimed to the shortage of traditional method was influenced by different degrees of saturation and each index could contain less vertical information in general. The paper choose the longhui district of hunan province in china as the study area, Studied the theory and method of using airborne LiDAR data to inversed forest LAI, established the optimum model of LAI inversion, propose a new approach for LAI inversion based on Lidar data. Finally relevant flight experiment and LAI inversion processing are introduced. The result indicate that the method is reliable, shows high accuracy inversion LAI with $\left(\mathrm{R}^{2}=0.878\right.$, $\left.\mathrm{RMSE}=0.223\right)$, compared with the TM images $\left(\mathrm{R}^{2}=0.706\right.$, RMSE $=0.430$ ), it verified the feasibility and validity of this proposed method in inversion of forest LAI for Environmental assessment.
\end{abstract}

Keywords: Leaf area Index, LiDAR, Forest Structural Parameters, Algorithm, DEM.

\section{INTRODUCTION}

Many researchers have conducted studies on Leaf area index (LAI) trends of different ecosystems and their relationships to climate change at various methods. Remote sensing technology is becoming more widely used because it is very efficient and provides high spatial and temporal resolution. However, in areas of high vegetation cover, measurements of this index will increase without limit [1-3]. Tucker proposed a normalized difference vegetation index (NDVI) that varies between -1 and 1 . The NDVI exploits the contrast in reflectance between the infrared and red portions of the electromagnetic spectrum and is well correlated to leaf area index (LAI), chlorophyll abundance, absorption of photosynthetically active radiation and gross primary production (GPP) quantity for Environmental assessment [4, 5].

LAI is very a critical parameter in process-based models of vegetation canopy response to global environmental change, Many researchers have conducted studies on LAI trends of different ecosystems and their relationships to climate change at various methods. The traditional methods of forest parameters measurement have some limitations in large scale [6-8]. However, due to the direct measurement capability with high precision on the forest vertical structure, Lidar technology shows unsurpassed advantages in the measurement of forestry ecological parameters.

Light Detection and Ranging (LiDAR) is an active remote sensing technology, which can determine the distance between the sensor and the target by emitting laser from sensors $[9,10]$. It has been applied to forestry research in many fields. Early researches find that canopy closure is most strongly related to the penetration capability of the laser pulse, and indicates that the pulsed laser system may be used to remotely sense the vertical forest canopy profile and assess tree height. Many work expose the enormous potential of laser applications in the forestry, which demonstrate that forest variables such as tree height, basal area, biomass, etc. can be estimated accurately using airborne laser scanner data (Nasset, 1997; Nelson, et al., 1997; Popescu, et al., 2002; Studies based on small footprint, high sampling density LiDAR data at individual tree level are reported later $[11,12]$. However, some studies show that the relationships between forest biophysical properties and airborne laser scanner data are different for the different geographical location, species composition, site quality, etc. In the case that remote sensing becoming more popular, the LiDAR technology has increasingly being used in the forestry as a new means of remote sensing. Many researchers have focused on LAI estimation using LiDAR data and explored the method of LAI retrieval based on LiDAR [13-16]. Although researchers have already worked out some retrieval algorithms of LAI, there is still a long way to go, for instance, retrieval accuracy and adaptability by single remote sensing sensor data. As a hotpot remote sensing technology, remote sensing has been proved great potential on LAI inversion $[17,18]$.

\section{METHOD}

Due to discrete items for Lidar data, it is needed to combine grid remote sensing data for inversion of the forest parameters in regional scale. Lefsky, et al. (2001) pointed out 
that in spite of the laser radar being sample data, it will greatly enhance the forestry measurement by integrating other grid remote sensing data owing to the very high precision. At present, combining Lidar data with optical, multiangular and microwave remote sensing data is an effective inversion way of canopy height and biomass in regional scale.

\subsection{Noise Removal of Lidar Data}

Point cloud data is helpful to detect and recognize surface features. However, during the process of point cloud data acquisition, due to the limitation of laser scanning device, original LiDAR point clouds usually contain system errors, which will result in the introduction of a large number of noises, So it is detrimental for the subsequent detection and classification recognition, and need to remove the noise points before classification processing, Noisy points can be divided into three categories according to their threedimensional characteristics: isolated points are these points that no other point cloud points in their neighborhoods Fig. (1). Extreme high points, the LiDAR points far higher from the nearby rough surface, usually caused by reflections of birds, low altitude airships or planes. Extreme low points, which are obviously lower than their adjacent ground LiDAR points, are caused by multiple reflections of laser pulse or system errors. The existence of extreme low points can lead to significant errors when interpolating DEM or DSM, hence affect the accuracy of objects classification. Based on height texture image Nardinocchi (2005) eliminated noise points by grid interpolation from (TIN). The main strategy of this approach is to classify point clouds by some specific techniques, its main advantage of this method is remove all kinds of noise naturally, while the main drawback is difficult to separate small construction from noise regions.

Finite-element reasoning (FEA) is introduced to eliminate noise, which its basic principle is that a complex object will be divided into several simple units, The threedimensional structure of hexahedron is regarded as the basic finite unit.it is described as Fig. (3), The topology relationship of adjacency unit can be easily achieved with this structure. the processing method of finite-element reasoning could be regarded as an extension of region growing in twodimensional raster image. By means of hexahedral mesh for subdivision of three-dimensional point cloud,.It is great useful of FEA reasoning for noise removal. Some agreements could be declared before algorithm description. Many experiment have showed that this method can detect noise points automatically, significantly lower risk of miscarriage of points.

\subsection{Airborne LiDAR Data Processing}

LiDAR data were collected on June 19, 2011 by Leica ASII-50 (density of point cloud is about 15 points every square metre), Which operated at near-infrared spectrum with first return record and last return record, the average sampling distance among footprints is approximately $0.6 \mathrm{~m}$, The principle of LiDAR Altimeter Waveform is shown in Fig. (2). Lidar point cloud data uses WGS84 coordinate system, and a universal transverse Mercator (UTM) projection,

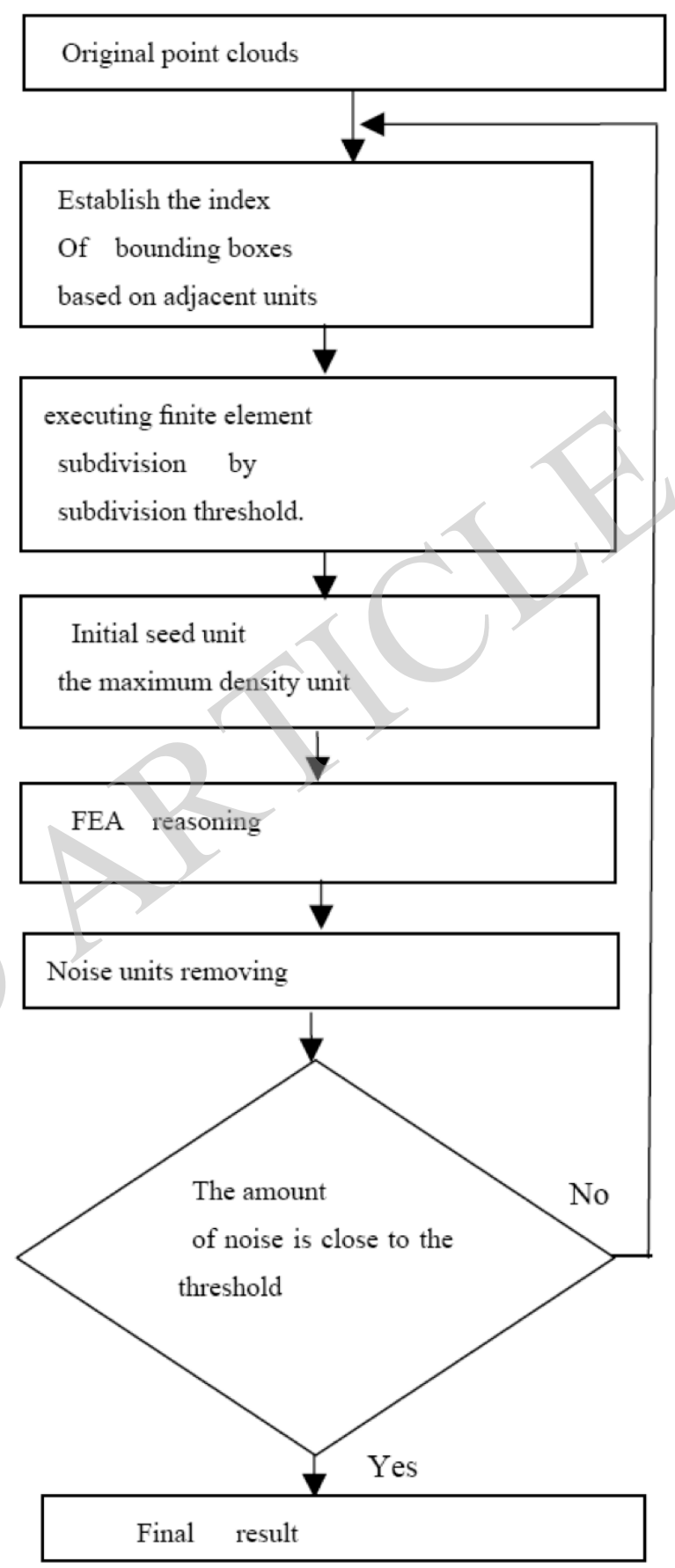

Fig. (1). The flow chart of noise removal.

the flight altitude is $600 \mathrm{~m}$ above the ground and the footprint diameter is about $0.35 \mathrm{~m}$, which is small so that the pulse can easily get through some sparse farmland and completely reach the ground. In this paper, both point cloud and full waveform data are used. For point cloud, the system records three types of echo, first echo, last echo and single echo, with point density of 0.95 points per square meter. The full waveform data contains information about the scanning angle, time interval between launch wave and reflection wave. Raw data contains a large number of gross errors or 


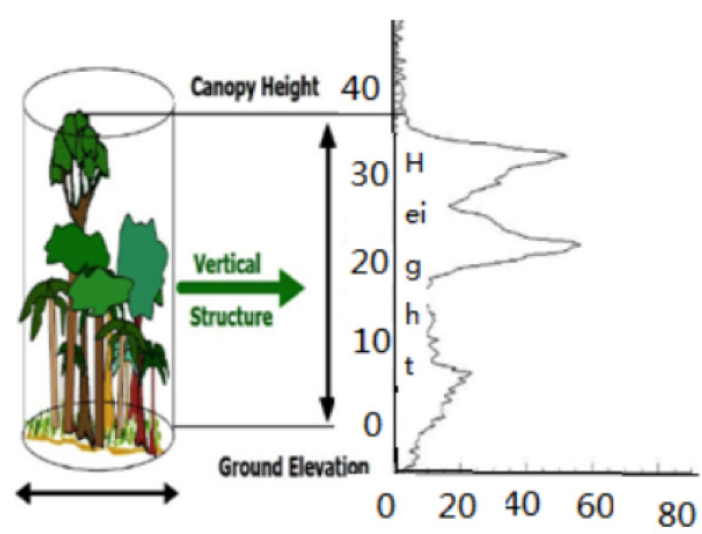

Fig. (2). The principle of Lidar Altimeter Waveform.

irrelevant information, such as data produced by local terrain mutation, reflected signal resulted from flying birds or other moving objects, or other local empty formed by no echo. In the data pre-processing,the data process software provided by equipment supplier should use artificial interactive editing to ensure the accuracy of terrain model and vegetation model generated later. The GPS carried by aircraft and DGPS on the ground positioned in real time during the flight for unifying and Images are another data source that we can employ,It provides information about objects contours and layout, which will improve the visualization effects. Image data is QuickBird,including 4 multi-spectral bands (nadir resolution is $2.44 \mathrm{~m}$ ) and a panchromatic band (nadir resolution is $0.61 \mathrm{~m}$ ). In our approach, we first locate a region of interest (ROI) from LiDAR data where an ROI contains candidate points. An ROI is then divided into separate objects using cue lines and GLCM textures from the visual data. Finally, via model matching and texture mapping, we obtain realistic renderings of geometric models of objects, even small ones, which closely correspond to the LiDAR elevation data.Data processing flow is shown in Fig. (3). Coordinate has been provided in UTM projection with an absolute accuracy of $<0.7 \mathrm{~m}$ in the $\mathrm{x}$ and $\mathrm{y}$ directions, and $<0.20 \mathrm{~m}$ in the $\mathrm{z}$ direction. The resolution along flight line is about $1.5 \mathrm{~m}$, the maximum resolution along scan line direction is about $0.07 \mathrm{~m}$, the minimum distance between buildings is $2.5 \mathrm{~m}$, the minimum height difference between terrain points and object points is $3.5 \mathrm{~m}$. Considering the design of the laser firing position, which is as high as the line of sight the laser altimeter reduces measurement errors as well as ensures a high measurement accuracy of $\pm 0.3 \mathrm{~m}$ within $300 \mathrm{~m}$ and an angle measurement accuracy of \pm 0.25 .

\subsection{Retrieval of LAI}

Point cloud intensity is influenced by many factors, such as scanning zenith angle, reflectance of the target, targetsensor distance and atmospheric condition. Nevertheless, when the points are gained by the same flight, the moisture content and roughness of soil, and even the atmospheric condition are very similar. According to Wagner (2006), the received energy can be specified by:

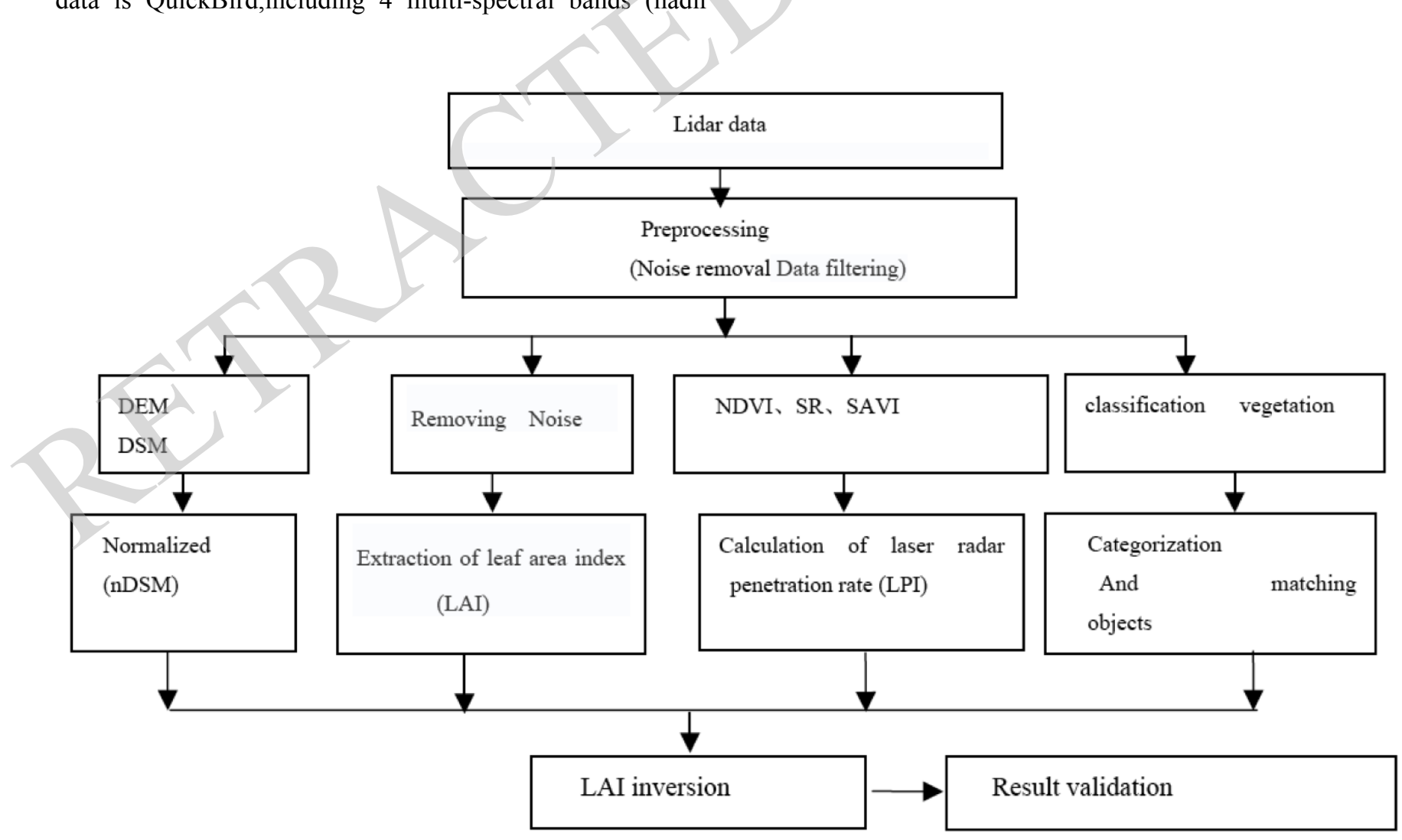

Fig. (3). Data processing flow chart. 
$E r=\frac{E_{t} D_{r}^{2} \rho}{4 \pi R^{4} \beta_{t}^{2}} A_{S} k_{1} k_{2}$

where Er and $E_{t}$ are the received and transmitted laser energy respectively, $\mathrm{R}$ stands for the sensor-target distance, $\beta_{\mathrm{t}}$ stands for the laser-beam divergence, $D_{r}$ stands for the diameter of the receiver aperture, $\rho$ stands for the reflectance of the target surface, $\mathrm{k}_{1}$ and $\mathrm{k}_{2}$ stands for the system and atmospheric transmission factors, respectively, and $\mathrm{A}_{\mathrm{s}}$ the illuminated target area.

(1) The vertical structural information obtained by LiDAR is used to mask images with stratification according to the measured tree species height statistics.the process helps in removing the interferences of forest gaps and other non-forest pixels as well as in extracting the canopy subsets. In addition, training samples can be easily extracted at a certain tree height of different species in the spectrum space. Therefore, based on the canopy subsets as well as the combined spectral differential and curve matching techniques, an automatic extraction method of training samples is realized. TerraScan offers the function model for classifying the ground points. The algorithm principle firstly chooses some low points, according to the area of max building to serve them as land surface points. And then, it repeatedly builds the triangle-net to gain the ground points (Guan \& Li, 2009). After gaining the ground points, it uses the GPS software to generate and interpolate the ground points into $1 \mathrm{~m}$ resolution DEM (Fig. 4).

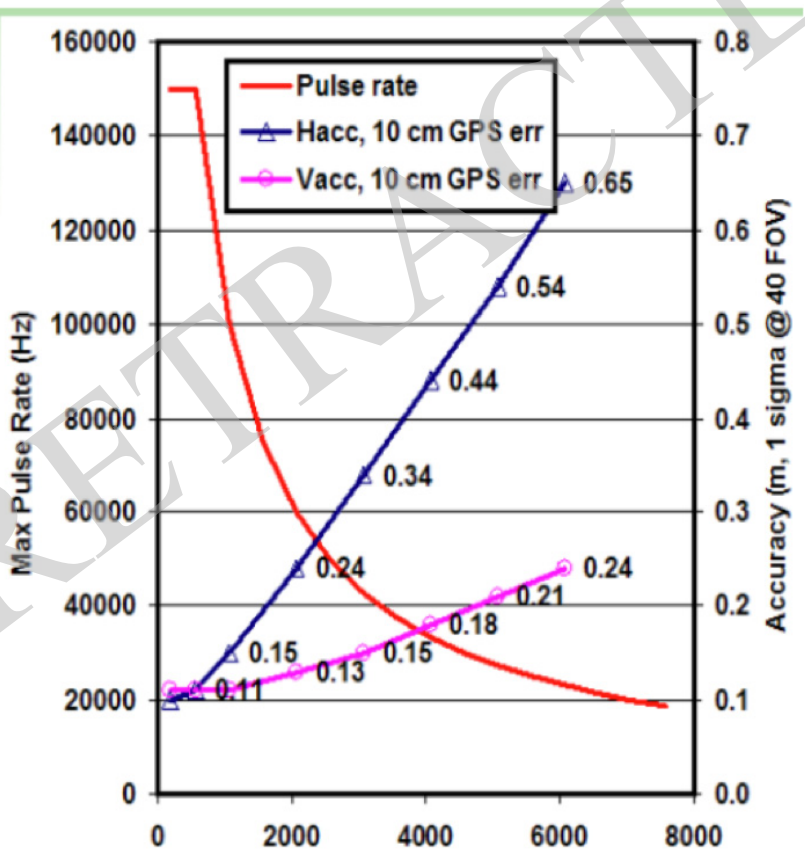

Fig. (4). Generate the ground points according to GPS.

(2) In order to evaluate the precision of leaf area index (LAI), reflectance data were collected with ASD spectroradiometer $(350 \sim 1050 \mathrm{~nm})$ in 2011 at two sampling sites, vegetation indices (NDVI, SAVI, SR) were ap- plied to regress against LAI, canopy reflectances were transformed with wavelet analysis, and extracted wavelet energy coefficient were applied in regression model for LAI estimation. transforming the point cloud into grid,when there have multiple points, using the max value as the pixelvalue. When the pixel value equals to 0 , using the average valuearound the pixel interpolates the grid data. And then getting $1 \mathrm{~m}$ resolution DSM.

(3) Generating the canopy height model (CHM) by DSM minus DEM. For a $5 \times 5$ window, if the central pixel is the minimum, let the pixel value equal to the average of the window. Furthermore, according to prior knowledge, if the CHM is less than $2.5 \mathrm{~m}$, the points in the grid are the points. And then, we can get the Lidar points in the forestland.it divided the study area into 5 $\mathrm{m} \times 5 \mathrm{~m}$ grids, where there may be a number of $(\mathrm{n})$ ground echoes, and the gap fraction $\left(f_{\text {gap }}\right)$ one grid then can be approximated by:

$$
f_{\text {gap }}=\frac{1}{n} \sum_{j=1}^{n} \frac{I_{g} \times R_{g}^{4}}{I_{\max } R_{\max }^{4}}
$$

where $I_{g}$ and $I_{\max }$ represent the intensity, $n$ is the count of points in one grid, $I_{g}$ and $R_{g}$ are the gap fraction, the intensity and the sensor-target distance of the $\mathrm{j}$ th ground echo, respectively.

$$
L A I=-\frac{\ln \left(f_{\text {gap }}\right)}{\lambda 0 G} \mu
$$

(4) co-registration should first be performed.The DEM with a high resolution of $0.5 \mathrm{~m}$ was obtained using the ground point cloud data through a TIN interpolation, and then an ortho-rectification of data was conducted to eliminate the image deformation caused by the terrain. For the same spatial resolution of $2 \mathrm{~m}, 20$ points, which were located along the road or flat area, were se-using the TerraScan to extract the ground points in the corn field. Calculating the value for every point. According to the longitude and latitude, the indensity and sensor-target distance $\mathrm{R}$ of every point can be gained. As usual, the points in the middle of the classification results can be considered as the pure pixels, considering the maximum likelihood classification's accuracy and features. For the huge number of points, we choose the first one hundred points. The probability of photons directly reaching the ground represents canopy transmittance:

where $\lambda_{0}$ is the Nilson parameter considering vegetation clumping effect, $\mathrm{G}$ is the mean projection of a unit of leaf area into the plane perpendicular to the incident laser direction, $\mu$ is the cosine of the zenith angle.

$$
T_{0}=e^{-\lambda_{0} \frac{G}{\mu} L A I}
$$

Canopy transmittance $\left(\mathrm{T}_{0}\right)$ equals to the gap fraction .Where $\mu$ can be calculated by the scanning angle. $G$ can be inferred from the prior knowledge, Accordingly, LAI can be calculated as follow: 


$$
f_{\text {gap }}=e^{-\lambda_{0} \frac{G}{\mu} L A I}
$$

\section{RESULT AND ANALYSIS}

\subsection{Study Site}

The experimental region is the longhui country of hunan Province in china, The site is surrounded by mountains around valley, and mainly influenced by the climate in highaltitude cloud circulation There are many crops and forest distributed on the irrigated oasis, which is on the alluvial fan and alluvial plain in the middle river. The main landscape type is mountain and hills, and mountainous area accounts for $49 \%$, the hilly area accounts for $28 \%$, plain and hummock (include waters) accounts for the remain 23\%. Four forest (Tropical evergreen broadleaved forest, Temperate Zone deciduous broad-leaved forest, Frigid Zone coniferous forest and mingled forests and shrubbery) fields on the flight were selected as the sampling fields, Method for LAI acquisition uses the Yao's method (Yao, et al., 2010). The DSM of laser point cloud in study area is shown in Fig. (5). According the forest growth, we chose the sample from the other fields with similar growth conditions, and then measured the leaf area by SunScan to determine LAI Each sample has precise GPS positioning data. SunScan does not wait for the special weather conditions and it can work properly in most light conditions. Hand-held terminal PDA is simple with large storage, which can meet the needs of large data measurement. Since the sensor using a wireless transmitting and receiving technology, it makes the measurement more convenient, quick access to the large area of forest region LAI. Wireless transmission range $250 \mathrm{~m}$, the vegetation coverage of 100 to $200 \mathrm{~m}$, greatly improve the efficiency of forest LAI.

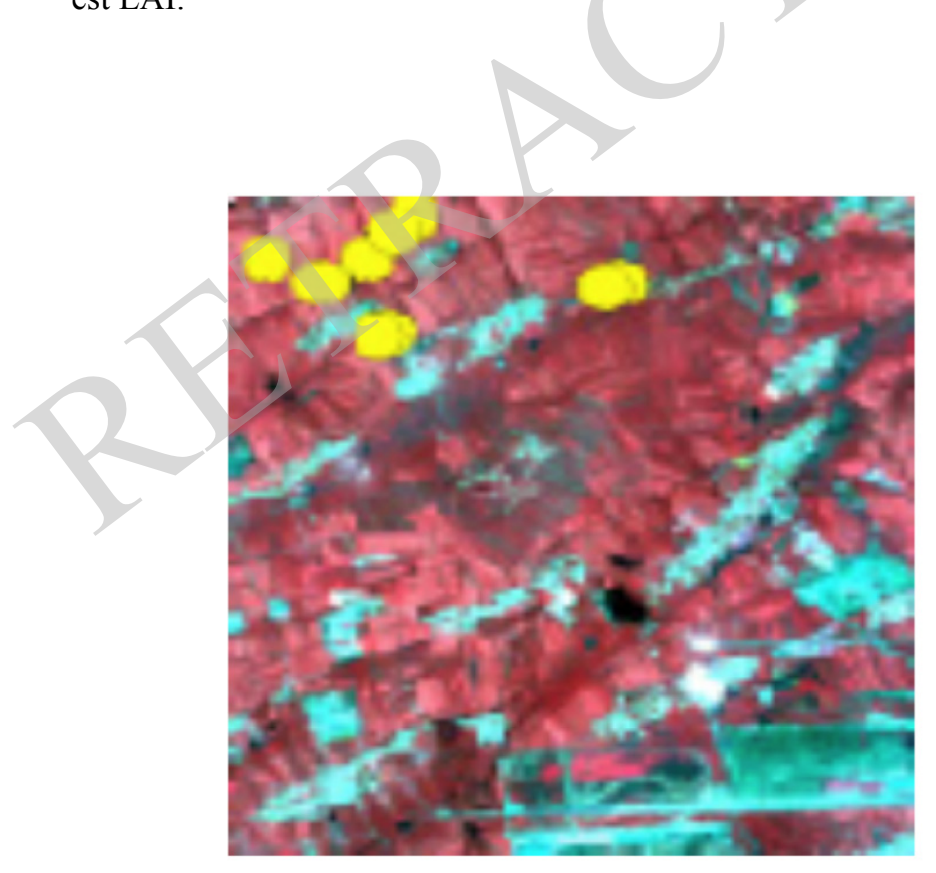

\subsection{Inversion Result of LAI}

In this study, a few forest variables were calculated based on the investigated data, containing basal area, mean height weighted by basal area, stem wood, live branch and foliage biomass were calculated using species allometric equations. the sampling radius of inversion for LAI is $10 \mathrm{~m}$. Then the data filtering, extraction of digital terrain model from the point cloud (DTM), and then from the digital surface model (DSM) for eliminating the effect of DTM, get normalized digital surface model (nDSM). Data classification and extraction of vegetation and ground point under different scales of a number of ground points and vegetation points. Finally calculate the laser radar penetration index and inversion calculation on LAI. The sampling data, LPI were calculated for each sample under various scales, LPI is the index of laser penetration:Where $\mathrm{L}$ stand for the leaf area index, I stands for the canopy light, $\mathrm{I}_{0}$ is above the canopy light, $\mathrm{k}$ is the extinction coefficient, depending on the direction of the leaf inclination Angle and the beam of light, light beneath the canopy is available. LPI formula instead of $\mathrm{I} / \mathrm{I}_{0},(1 / \mathrm{LPI})$ containing the intercept of the regression analysis with the measured LAI, inversion model based on airborne LiDAR data. This research adopts the different radius of the circular laser point cloud data resampling, sampling data are calculated respectively in different space scale of LPI, and carries on the regression analysis and the measured LAI, calculate model of the correlation coefficient $\left(\mathrm{R}^{2}\right)$ and root mean square error (RMSE), to determine the optimal sampling radius of the model with a radius of $5 \mathrm{~m}, 10 \mathrm{~m}, 15 \mathrm{~m}, 20 \mathrm{~m}$ and $25 \mathrm{~m}$. The accuracy of LAI with five spatial scales based on echoes counts (Table 1). From Table 1, it is clear that the fractional cover of Lidar and field data agree well, and only the 4 plot has a relative large error. The reason is that the tiny difference of soil condition and the scanning angle may cause different soil BRDF, resulting in the retrieval error. Through the forest coverage map, Results of LAI inversion is shown in Fig. (6), it can be seen that the inversion result of forest coverage was consistent with the vegetation

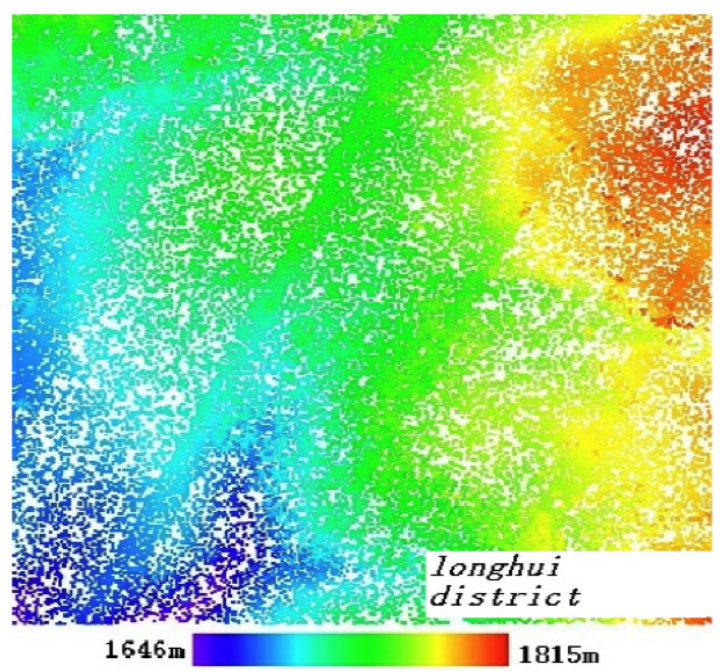

Fig. (5). The DSM of laser point cloud in study area. 
Table 1. The accuracy of LAI with five spatial scales based on echoes counts.

\begin{tabular}{|c|c|c|c|c|c|}
\hline Radius & $\mathbf{5 m}$ & $\mathbf{1 0 m}$ & $\mathbf{1 5 m}$ & $\mathbf{2 0 m}$ & 0.739 \\
\hline \hline $\mathrm{R}^{2}$ & 0.678 & 0.832 & 0.723 & 0.702 & 0.674 \\
\hline Adjust $\mathrm{R}^{2}$ & 0.785 & 0.796 & 0.744 & 0.247 & 0.750 \\
\hline RMSE & 0.245 & 0.238 & 0.248 & $<0.001$ & $<.269$ \\
\hline P-VALUE & $<0.001$ & $<0.001$ & $<0.001$ & $<0.001$ \\
\hline
\end{tabular}

Note: $\mathrm{R}^{2}$ - coefficient of determination, Adjust $\mathrm{R}^{2}$ - adjust $\mathrm{R}^{2}$, RMSE-the root mean square error

Table 2. Comparisons of LAI between those derived from Lidar and field measurements for the four fields.

\begin{tabular}{|c|c|c|c|c|c|c|}
\hline \multirow{2}{*}{ Plots } & \multicolumn{2}{|c|}{ Lidar results } & \multicolumn{2}{c|}{ Field measurements } & \multirow{2}{*}{ Absolute error } & \multirow{2}{*}{ Relative error/\% } \\
\cline { 2 - 6 } & Average & Standard deviation & Average & Standard deviation & \\
\hline \hline 1 & 0.650 & 0.232 & 0.045 & 0.048 & 0.034 \\
\hline 2 & 0.630 & 0.030 & 0.625 & 0.000 & 0.005 & 0.459 \\
\hline 3 & 0.489 & 0.138 & 0.634 & 0.062 & 0.045 & 0.800 \\
\hline 4 & 0.543 & 0.024 & 0.458 & 0.035 & 0.055 & 8.265 \\
\hline
\end{tabular}

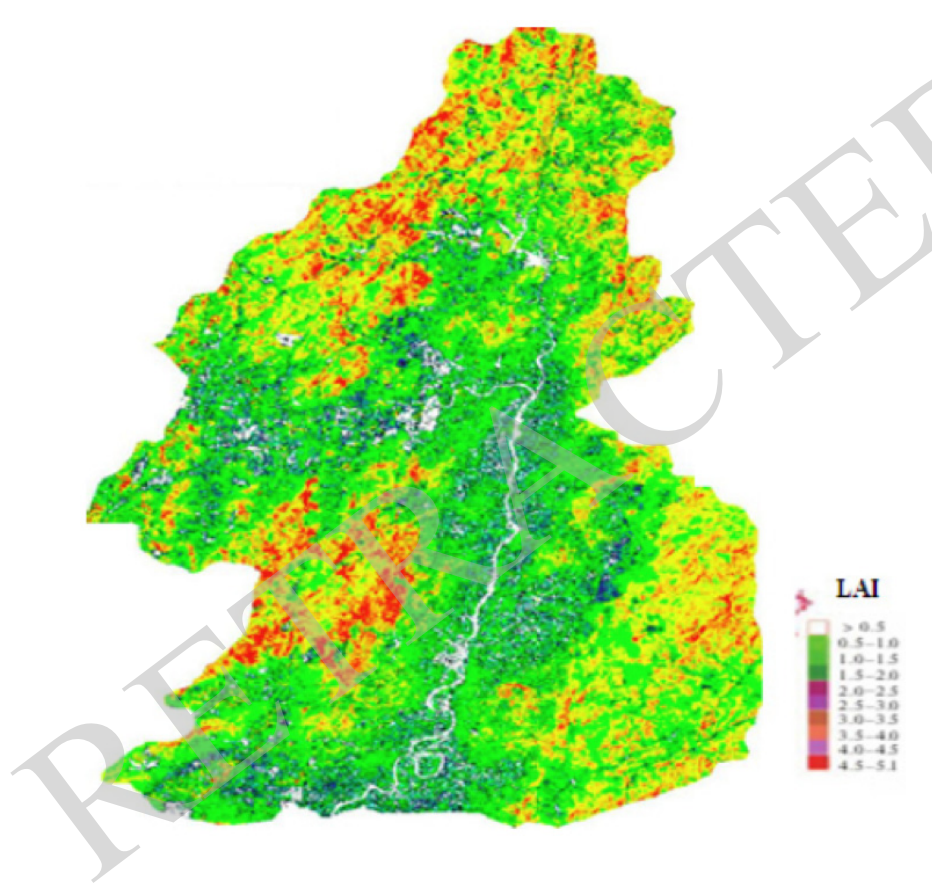

Fig. (6). The inversion map of LAI in the study area.

distribution in the study area. Comparisons of LAI between those derived from Lidar and field measurements (Table 2), it is showed that the LAI of Lidar and filed data agree well, and only that the $3^{\text {th }}$ plot has a relative large error. The retrieval error of fractional cover will transfer to LAI.

$$
L=-\frac{1}{k} \ln \left(\frac{I}{I_{0}}\right)
$$

Fitted with the measured coverage data, the value of $\mathrm{R}^{2}$ was 0.88 , and the error with sample data was about 0.07 0.08 . In addition, compared to the measured results by Huang (2010), which the forest coverage value in sample area was 0.54 , the difference value with the inversion results of forest coverage $(0.769) \mathrm{s}$ was only 0.056 . Results show that based on airborne and spaceborne LiDAR data LAI inversion accuracy is higher than that of the corresponding optical remote sensing inversion accuracy.

LAI statistical result of the whole study area in showed in Fig. (7), It show that the accuracy of inversion LAI can be improved with increasing the number of observation angle.there are more than $86 \%$ retrieval points distributing in the range of field data, Apart from the classification error, the distribution is reasonable with the forest LAI distribution. The accuracy of LAI estimations may be influenced by cumulative errors. First, the measured errors of plot data acquisition are inevitable. Second, the scanning time of LIDAR data is at the turn of autumn and winter, not summer with dense foliage, so the echoes returned from leaves decreases. Plot coordinates errors and sampling density of LIDAR data could affect the laser height distribution, and ultimately affect the estimation accuracy. In addition, underestimation of tree height leads to the underestimation of the biomass directly. It can be seen that estimation precision of foliage biomass is relatively low. In addition, it is necessary to consider the impact of different geographical regions and forest types on the estimation of LAI Fig. (8).

In order to evaluate the reliability of Lidar inversion method, using leave-one-out cross-validation method, predicted LAI was evaluated by using 16 measured LAI data, it was showed that the determination coefficient is 0.878 , the root mean square error is 0.208 towards measured value and predicted values (Fig. 9), it found that the results of forest 


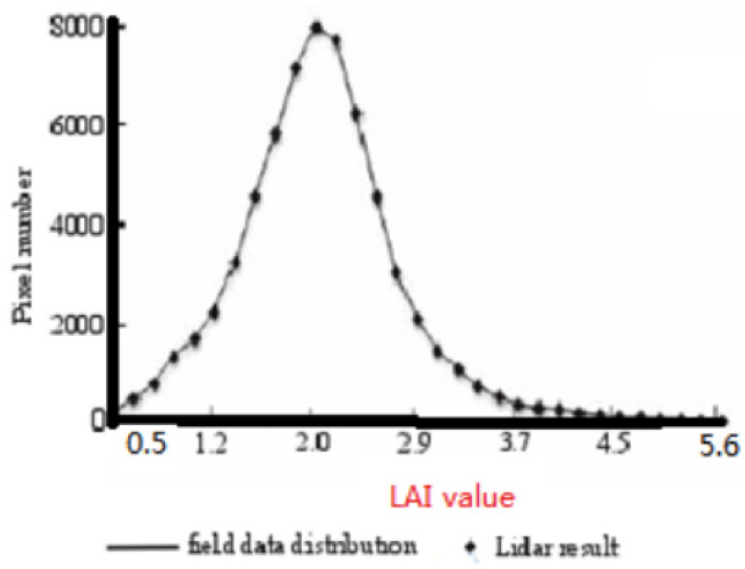

Fig. (7). LAI statistical result of the whole study area.

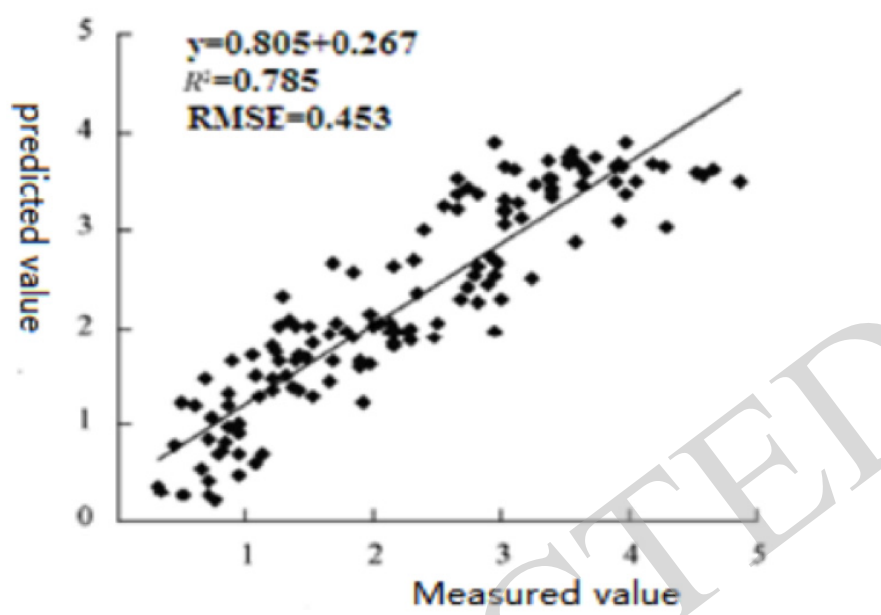

Inversion of L.Al from 1.31 data

Fig. (8). The inversion value of TM data.

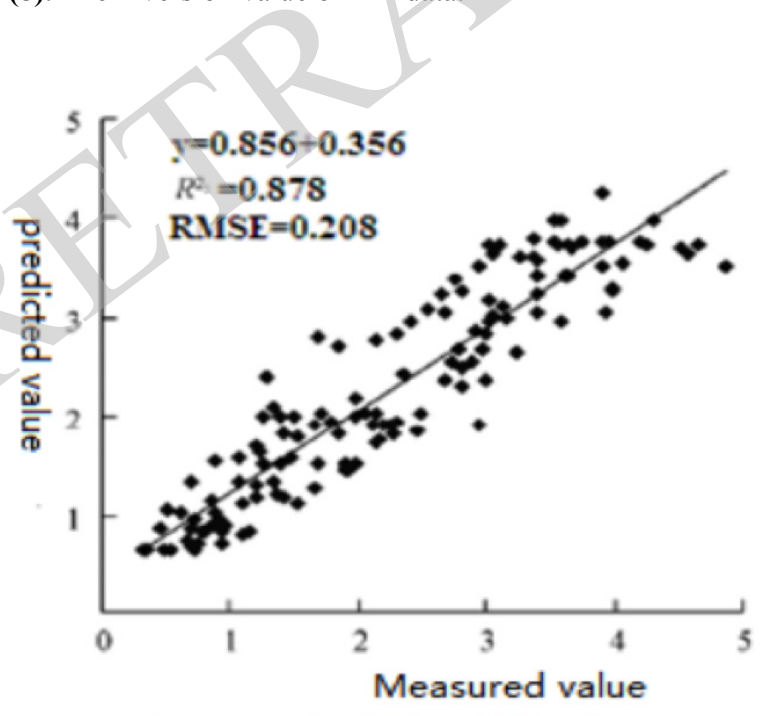

Inversion of LAI from Lidar data

Fig. (9). The inversion of value of Lidar data.
LAI joint inversion model was consistent with the distribution of forest type map. Hence, these results can be better applied to the quantitative inversion of other forest biophysics parameters. LiDAR data offer a new way to accurately estimate forest LAI. Especially, satellite borne LiDAR data open the possibility of global forest LAI estimation with high accuracy.

In order to enhance contrast TM data is also employed to inversion the same region. The result is showed in Fig. (8). it indicate that the method is reliable, shows high accuracy inversion LAI with $\left(\mathrm{R}^{2}=0.878, \mathrm{RMSE}=0.223\right)$, compared with the TM images $\left(\mathrm{R}^{2}=0.706\right.$, $\mathrm{RMSE}=0.430$, has a better fitting effect, and can input more band information to improve the leaf area index remote sensing inversion accuracy which is suitable for forest and the experiment also validates the great potential for Lidar to be applied to monitor forest structural parameters.

\section{CONCLUSION AND DISCUSSION}

As part of the biosphere, vegetation plays an important role in regulating the global carbon cycle. vegetation helps maintain climatic stability in many ways, including photosynthesis, evapotranspiration, modifying surface albedo and roughness. Light Detection And Ranging (LiDAR) is a new emerging active remote sensing technology in recent years, which has developed very rapidly in the world, Since leaf area index (LAI) is very often a critical parameter in processbased models of vegetation canopy response to global environmental change, for numerous studies of interaction of atmosphere and vegetation, rapid, reliable and objective estimations of LAI are essential.it hypothesize that adjusting key parameter can improve estimates and can compensate for structural errors caused by adopting the one-leaf strategy in the algorithm. The objective of this study is to evaluate capacity of parameter adjustment in the algorithm to improve estimates for multiple plant functional types. We used Lidar measurements to optimize key parameters in the algorithm. The accuracy of LAI estimation is closely related with the extraction of laser height. Airborne laser system samples discretely, the sampling points have a certain interval. It may miss the top of tree easily, resulting in height underestimation. The weighted mean height algorithm also makes large trees influencing the mean height of plots than small ones, and there is no connection with species. However, increasing sampling accuracy can improve the precision of LAI estimation, but it compensates for excessively high costs. On the other hand, ground points used to generate digital elevation model (DEM) may be covered in the dense forest, causing height errors of canopy height model (CHM). It investigate the accuracy of digital terrain models (DTM) for four different stand density forests and the results show that the greater DTM errors can cause greater canopy density. Results show that retrieving LAI with high accuracy and resolution is possible using the Lidar data. In the future, Lidar will have extensive applications for precision agriculture and other agricultural production..As spatial distribution patterns were concerned, the results of LAI height by joint inversion model was consistent with the distribution of forest type map. Hence, these results can be better applied to the quantitative inversion of other forest biophysics parameters. How to improve the accuracy of forest LAI inversion and make the 
inversion method simple and easy to use is a very important study area at home and abroad, LiDAR data offer a new way to estimate forest LAI with high accuracy for ecological environment research.

\section{CONFLICT OF INTEREST}

The authors confirm that this article content has no conflict of interest.

\section{ACKNOWLEDGEMENTS}

The authors would like to thank the anonymous reviewers for their careful reading of this paper and for their helpful comments, This work is supported by the Geographic Spatial Information Engineering Key Laboratory of the State Bureau of Surveying and mapping (No201215).

\section{REFERENCES}

[1] K. S. Lim and P. M. Treitz, "Estimation of above ground forest biomass from airborne discrete return laser scanner data using canopy-based quantile estimators," Scandinavian Journal of Forest Research, vol. 19, no. 6, pp. 558-570, 2004.

[2] K. F. Cao and J Chang, "The ecological effects of an unusual climatic disaster: the destruction to forest ecosystems by the extremely heavy glaze and snow storms occurred in early 2008 in southern China," Chinese Journal of Plant Ecology, vol. 34, no. 2, pp. 123124,2010

[3] E. Nasset, "Determination of mean tree height of forest stands using airborne laser scanner data," ISPRS Journal of Photogrammetry and Remote Sensing, vol. 52, no. 2, pp. 49-56, 1997.

[4] L. X. Dong, Estimation of the Forest Canopy Height and Above ground Biomass Based on Multiplicatel Remote Sensing in Three Gorges. Institute of Remote Sensing Applications Chinese Academy of Sciences (CAS), Beijing, pp. 56-98, 2008.

[5] L. X. Dong, B. F. Wu, and Z, H, Guo, "Estimation of forest canopy height by integrating multisensor data," In: Proceedings of the SPIE, 2009.

[6] Y. F. Bao, C. X. Cao, H. Zhang, E. X. Chen, Q. S. He, H. B. Huang, Z. Y. Li, X. W. Li and P. Gong, "Synchronous estimation of DTM and fractional vegetation cover in forested area from airborne LIDAR height and intensity data," Science in China Series E Technological Sciences, vol. 51, (Suppl. 2), pp. 176-187, 2008.
[7] G. B. Bonan, "Importance of leaf area index and forest type when estimating photosynthesis in boreal forests," Remote Sensing of Environment, vol. 43, no. 3, pp. 303-314, 1993.

[8] M. A. Lefsky, W. B. Cohen, S. A. Acker, G. G. Parker, T. A. Spies, and D. Harding, "LiDAR remote sensing of the canopy structure and biophysical properties of Douglas-fir western hemlock forests," Remote Sensing of Environment, vol. 70, no. 3, pp. 339-361, 1999.

[9] Q. Li and H. C. Ma, "The study of point-cloud production method based on waveform laser scanner data," Acta Geodaetica et Cartographica Sinica, vol. 37, no. 3, pp. 349-354, 2008.

[10] J. E. Means, S. A. Acker, D. J. Harding, J. B. Blair, M. A. Lefsky, W. B. Cohen, M. E. Harmon and W. A. McKee, "Use of largefootprint scanning airborne lidar to estimate forest stand characteristics in the Western Cascades of Oregon," Remote Sensing of Environment, vol. 67, no. 3, pp. 298-308, 2005.

[11] J. S. Chen H. Lin and Z. Y. Pei, "Application of ENVISAT ASA data in mapping rice crop growth in Southern China," IEEE Geoscience and Remote Sensing Letters, vol. 4, no. 3, pp. 431-435, 2007.

[12] Q. Cheng, J. F. Huang. C. Wang, and Y. L. Tang, "Analyses of the correlation between rice LAI and simulated MODIS vegetation indices, red edge position," Transactions of the Chinese Society of Agricultural Engineering, vol. 19, no. 5, pp. 104-108, 2013.

[13] J. G. P. W. Clevers, "Application of a weighted infrared-red vegetation index for estimating leaf area index by correcting for soil moisture," Remote Sensing of Environment, vol. 29, no. 1, pp. 2537, 2012 .

[14] Z. Q. Yin J. F. Guan X. H. Zhang, and C. M. Cao, "The Monte Carlo method and its application," Physics and Engineering, vol. 12 , no. 3, pp. 45-49, 2002.

[15] J. X. Zhang, and W. Su, "Sensitivity analysis of ES-Wheat model parameters based on EFAST method," Journal of China Agricultural University, vol. 17, no. 5, pp. 149-154, 2012.

[16] Y. Zhang Q. Y. Meng J. L. Wu, and F. Zhao, "Study of environmental vegetation index based on Environment Satellite CCD data and LAI inversion," Spectroscopy and Spectral Analysis, vol. 31, no. 10, pp. 2789-2793, 2011.

[17] F. Deng J. M. Chen S. Plummer and M. Chen, "Algorithm for global leaf area index retrieval using satellite imagery," IEEE Transactions on Geoscience and Remote Sensing, vol. 44, no. 8, pp. 2219-2229, 2006.

[18] R. Liu J. M. Chen J. Liu F. Deng, and R. Sun, "Application of a new leaf area index algorithm to China's landmass using MODIS data for carbon cycle research," Journal of Environmental Management, vol. 85, no. 3, pp. 649-658, 2007.

Received: September 16, 2014

Revised: December 23, 2014

Accepted: December 31, 2014

(C) Zuowei et al.; Licensee Bentham Open.

This is an open access article licensed under the terms of the Creative Commons Attribution Non-Commercial License (http://creativecommons.org/licenses/by-nc/3.0/) which permits unrestricted, non-commercial use, distribution and reproduction in any medium, provided the work is properly cited. 